\title{
Prognostische Relevanz der Metastasenlokalisation bei nicht kleinzelligem Bronchialkarzinom im Tumorstadium IV - Ergebnisse einer prospektiven epidemiologischen Studie
}

\author{
Prognostic Relevance of the Metastatic Localisation in Patients with Stage IV NSCLC - Results of a Prospective \\ Epidemiological Study
}

Autoren

Institute
T. Blankenburg ${ }^{1}$, B. Gottschlich ${ }^{1}$, W. Schütte' ${ }^{1}$, H. Neef ${ }^{3}$, J. Haerting ${ }^{2}$

Krankenhaus Martha-Maria Halle-Dölau, Klinik für Innere Medizin II (Chefarzt PD Dr. W. Schütte)

2 Institut für Medizinische Epidemiologie, Biometrie und Informatik der Martin Luther Universität Halle-Wittenberg

(Direktor: Prof. J. Haerting)

3 Tumorzentrum der Martin Luther Universität Halle-Wittenberg (Vorsitzender: Prof. H. Schmoll) eingereicht 14.2.2010

akzeptiert nach Revision 4. 5.2010

\section{Bibliografie}

DOI http://dx.doi.org/

10.1055/s-0029-1244238

Online-Publikation: 24. 6. 2010

Pneumologie 2010; 64:

679-685 @ Georg Thieme

Verlag KG Stuttgart · New York

ISSN 0934-8387

\section{Korrespondenzadresse}

Dr. med. Thomas Blankenburg

Krankenhaus Martha-Maria

Halle-Dölau

Klinik für Innere Medizin II

Röntgenstraße 1

06120 Halle

th.blankenburg@gmx.de

\section{Zusammenfassung \\ $\nabla$}

Patienten mit nicht kleinzelligem Bronchialkarzinom sind häufig bei Diagnosestellung im metastasierten Tumorstadium. Das Überleben ist in dieser Situation ungünstig, diese Patienten können von Chemotherapie profitieren. Hauptziel dieser Arbeit ist es, den prognostischen Einfluss der Metastasenlokalisation an unselektionierten Patienten zu untersuchen. Das zweite Ziel dieser Arbeit besteht in der versorgungsepidemiologischen Analyse klinischer Faktoren, die einen Einfluss auf die Therapieentscheidung bei metastasiertem NSCLC haben könnten.

Klinische Daten sowie Überlebensdaten wurden von 336 inzidenten Patienten mit metastasiertem NSCLC einer definierten Region erfasst. Die Rekrutierungsdauer betrug 31/2 Jahre, die mindeste Nachbeobachtungsdauer 24 Monate. Diese Untersuchung ist Teil der HALLUCA Studie, die vom Bundesministerium für Gesundheit gefördert wurde.

Das mediane und 1-Jahres-Überleben dieser Patienten war, in Abhängigkeit von der Metastasenlokalisation, signifikant unterschiedlich und variierte zwischen 2,2 Monaten resp. 4,7\% (Lebermetastasen) und 11 Monaten resp. 44,5\% (Lungenmetastasen). Der Einfluss der Metastasenlokalisation auf das Überleben bestätigte sich in der multivariaten Analyse. Der Anteil an chemotherapierten Patienten an allen metastasierten Patienten betrug 39\%. Patienten mit Leber-, Knochen- und multiplen Metastasen erhielten signifikant seltener Chemotherapie, obwohl im Mittel der Leistungszustand nicht schlechter war als in den anderen Gruppen.

Mit der vorliegenden populationsbezogenen versorgungsepidemiologischen Studie kann eine prognostische Heterogenität der Patienten mit metastasiertem NSCLC belegt werden. Dies sollte berücksichtigt werden für die Stratifizierung klinischer Studien bei diesen Patienten. Die Art der

\section{Abstract \\ $\nabla$}

Patients with non-small cell lung cancer (NSCLC) frequently suffer from stage IV disease at the time of presentation. Survival of these patients is disadvantageous although they may benefit from chemotherapy. The main purpose of this investigation was to evaluate the prognostic relevance of the metastatic localisation in unselected patients. The second purpose was to evaluate the impact of clinical characteristics on the kind of decision-making in patients with stage IV NSCLC in an epidemiological manner.

Clinical data as well as survival of 336 patients with stage IV NSCLC were analysed. The recruitment period was $3 \frac{1}{2}$ years, mean follow-up was 24 months. This investigation was part of the HALLUCA studies which were sponsored by the German Ministry of Health.

Localisation-dependent median and 1-year survivals were significantly different and varied between 2.2 months and $4.7 \%$ (liver metastases) and 11.0 months and $44.5 \%$ (lung metastases). The different survival remained significant in the multivariate analyses with age, performance status, treatment and histology as co variables. The chemotherapy rate of all patients with stage IV NSCLC was $39 \%$. Patients with liver, bone and multiple metastases received less often chemotherapy compared to patients with other metastases although the performance status was not different to the other groups.

Although there are some limitations in this investigation, these epidemiological data demonstrate the prognostic heterogeneity of stage IV NSCLC patients which should be considered for stratification in controlled clinical trials. Regional treatment decision-making is different from guidelines and controlled clinical trials. Further regionally orientated trials are necessary to improve the transformation from clinical trials to regional medical care. 
Therapieentscheidung in der Region unterscheidet sich von den Empfehlungen und den Ergebnissen kontrollierter klinischer Studien. Weitere regional orientierte versorgungsepidemiologische Untersuchungen sind erforderlich, um den Durchsetzungsgrad wissenschaftlicher Forschungsergebnisse im klinischen Alltag zu verbessern.

\section{Einleitung}

\section{$\nabla$}

Das Bronchialkarzinom ist weltweit die häufigste Krebstodesursache, die Inzidenz wird mit 1,04 Millionen, die Mortalität mit 921000 Fällen pro Jahr angegeben [1,2]. Zum Zeitpunkt der Diagnose liegt bei mindestens $40 \%$ der Patienten mit nicht kleinzelligem Bronchialkarzinom (NSCLC) ein metastasiertes Stadium vor [3]. In diesem Stadium ist die Einjahresüberlebensrate aller Patienten mit zwischen 10 und 25\% schlecht [4]. Unter Chemotherapie bessert sich die Einjahresüberlebensrate, ist aber mit 31 $40 \%$ ungünstig [5]. Aus diesem Grund sind prädiktive bzw. prognostische Parameter, die das Ansprechen auf Chemotherapie bzw. die Überlebenswahrscheinlichkeit differenzieren, wünschenswert. Während prädiktive Faktoren einen bestimmten Befund mit einer gewissen Wahrscheinlichkeit vorhersagen (z.B. Ansprechen auf Chemotherapie), wird von prognostischen Faktoren gesprochen, wenn Faktoren beschrieben werden, die mit der Vorhersage eines Endzustandes assoziiert sind (Überleben).

Bekannte ungünstige prognostische Faktoren für das Gesamtüberleben bei Lungenkrebs sind höheres Lebensalter und männliches Geschlecht [6]. In einer retrospektiven Analyse aus dem Jahre 2004 hatte der aktuelle Raucherstatus prognostische Relevanz [27]. Ungünstige prognostische Bedeutung haben im metastasierten Stadium neben dem histologischen Typ eine Gewichtsabnahme sowie ein reduzierter klinischer Leistungszustand [12,13].

Prädiktiven Wert z.B. für die Effektivität der Chemotherapie können immunhistochemische Befunde haben. Außerdem konnte eine randomisierte klinische Studie zeigen, dass Patienten mit Adeno- oder großzelligem NSCLC signifikant besser von einer Pemetrexed-Platin-Chemotherapiekombination profitieren als Patienten mit Plattenepithelkarzinom [7]. Eine direkte Chemotherapiesensitivitätstestung ist jedoch noch nicht klinisch relevant [8].

In epidemiologischen Untersuchungen kann die Behandlungsrealität eines unselektionierten Patientenkollektivs regionsbezogen abgebildet werden $[9,10]$. Diese Analysen zeigen den Durchsetzungsgrad von Studienergebnissen und Behandlungsempfehlungen und können prognostische Aussagen aus einem unselektionierten Krankheitsgut generieren, die unter Umständen in kontrollierten klinischen Studien bisher nicht betrachtet worden sind. Der Vorteil eines versorgungsepidemiologischen Ansatzes liegt in der Abbildung der aktuellen regionsbezogenen Behandlungsrealität, der Nachteil dieser Studienform besteht in einer geringeren Datendichte.

Vor diesem Hintergrund ergibt sich für die vorliegende Arbeit die folgende Hauptfragestellung: Hat die Metastasenlokalisation bei unselektionierten Patienten mit nicht kleinzelligem Bronchialkarzinom im Tumorstadium IV eine prognostische Relevanz? Der versorgungsepidemiologische Aspekt dieser Arbeit soll die Problematik der Art der Therapieentscheidung in Abhängigkeit von den erfassten klinischen Parametern als Marker für die Qualität der medizinischen Versorgung betrachten.

\section{Patienten und Methoden}

$\boldsymbol{\nabla}$

\section{Methodik}

Über insgesamt 31/2 Jahre wurden alle inzidenten Patienten, die an einem Bronchialkarzinom erkrankt waren, im Rahmen einer epidemiologischen Feldstudie (Hallesche Lungen-Carcinomstudie $=$ HALLUCA) erfasst und im Verlauf über mindestens 24 Monate nachbeobachtet.

Die Studienregion umfasste ein Areal von 1,5 Millionen Einwohnern. Prospektiv konnten alle klinischen und ambulanten Einrichtungen, die an der Betreuung von Patienten mit Bronchialkarzinom beteiligt waren, zur Studienteilnahme gewonnen werden. Insgesamt nahmen 25 Krankenhäuser sowie alle niedergelassenen Pneumologen, Onkologen und Strahlentherapeuten teil. Die Koordination erfolgte im Tumorzentrum Halle in Zusammenarbeit mit dem Institut für Medizinische Epidemiologie, Biometrie und Informatik der Martin-Luther-Universität Halle-Wittenberg durch eine interdisziplinäre Studienleitung, die aus Thoraxchirurgen, Pneumologen, Epidemiologen und Psychologen bestand.

\section{Sicherung der Datenqualität}

Aus Arztbriefen, deren Struktur vorgegeben war und überprüft wurde, wurden sowohl zum Diagnosezeitpunkt als auch zum Zeitpunkt der verschiedenen ambulanten und stationären Therapien sowie zur Nachsorge Daten erfasst. Durch Meldung der histologischen Befunde durch die Pathologien sowie der thoraxchirurgischen Abteilungen der Region konnte die Erfassungsrate erhöht werden. Fehlende Dokumentationen wurden aktiv durch das Studienzentrum bei dem entsprechenden Behandler eingeholt. Es erfolgte eine regelmäßige halbjährliche Analyse der Datenqualität und ein Qualitätsmanagement anhand der Akten mit den rekrutierenden Kliniken. Die Qualität der diagnostischen und therapeutischen Maßnahmen wurde in monatlichen Fallkonferenzen analysiert und in regional konsentierten Empfehlungen zur Diagnostik, Therapie und Nachsorge beschrieben.

Die Überlebenszeit wurde primär durch die entsprechenden Epikrisen erfasst. Daneben erfolgte eine Analyse aller Totenscheindaten der Region in jährlichen Intervallen sowie ein Datenabgleich der Totenscheindaten für alle erfassten Fälle mit dem Gemeinsamen Krebsregister der fünf neuen Bundesländer und Berlin. Erfasst wurde der Tod jeglicher Ursache.

\section{Studienpopulation}

Von insgesamt 1696 inzidenten Patienten mit Bronchialkarzinom hatten 72,1\% (1223) ein nicht kleinzelliges Bronchialkarzinom. Im Vergleich zur alters- und geschlechtskorrigierten geschätzten Inzidenz der Region wurde eine Erfassungsrate von 73,8\% erreicht. Der Anteil an Patienten mit metastasiertem nicht kleinzelligem Bronchialkarzinom lag bei 31,9\% (390 Patienten) aller Patienten mit NSCLC. Bei 339 der 390 Patienten mit metastasiertem $\operatorname{NSCLC}(86,9 \%)$ konnte diese Untersuchung durchgeführt werden.

\section{Sicherung der Metastasenlokalisation}

Routineuntersuchungen, die bei allen Patienten zur Metastasendetektion durchgeführt wurden, waren die thorakale Computertomografie mit Kontrastmittel sowie die abdominelle Ultraschalluntersuchung. Schädel-CT bzw. -MRT, abdominelle MRT, endoskopischer Ultraschall und Knochenszintigrafie wurden bei klinischem Verdacht auf Metastasierung oder abnormen paraklinischen Befunden durchgeführt. Bei unsicheren Befunden (z.B. unklarer Lungenrundherd, sonografisch nicht eindeutiger Be- 
fund) erfolgte eine histologische Klärung. Der Umfang und die Art der weiterführenden Diagnostik orientierten sich an der therapeutischen Relevanz. Es konnte sichergestellt werden, dass bei allen Patienten eine Schnittbilddiagnostik hinsichtlich aller folgenden Lokalisationen erfolgte: Lunge, Nebennieren, Hirn, Leber, Knochen. Die systematische Untersuchung aller anderen Metastasenlokalisationen erfolgte auch nach dem Nachweis einer Metastasierungslokalisation.

\section{Patientencharakteristik}

Die Patientencharakteristik ist in $\bullet$ Tab. 1 dargestellt.

$83 \%$ der Patienten waren männlich, 17\% weiblich. Die Männer waren etwa 2 Jahre älter als die Frauen. 50\% aller Patienten hatten einen guten Performancestatus, 33\% der Patienten hatten einen schlechten Performancestatus. Plattenepithelkarzinom trat doppelt so häufig auf wie Adenokarzinom. Großzelliges Karzinom wurde nur in 2\% der Fälle diagnostiziert. In 33\% der Fälle wurde nicht kleinzellige Histologie ohne weitere Spezifikation angegeben.

\section{Verteilung der Metastasenlokalisation}

Die Art der Metastasenlokalisation ist in $\bullet$ Tab. 2 dargestellt. $36 \%$ aller Patienten hatten mehrere Metastasenlokalisationen. In einem Viertel aller Patienten war die einzige Metastasenlokalisation die Lunge, die anderen Metastasenlokalisationen waren seltener.

Den Zusammenhang zwischen Lebensalter und Lokalisation beschreibt die Tab. 2. Auffällig ist, dass Patienten mit Hirnmetastasen im Mittel 58 Jahre alt waren, während Patienten mit Lebermetastasen im Mittel 70 Jahre alt waren. Die Patienten mit anderen Metastasenlokalisationen waren im Mittel 65 Jahre alt.

Die Häufigkeit des Befalls der verschiedenen Lokalisationen in Abhängigkeit von der Histologie beschreibt die Tab. 3. Auffällig ist, dass bei Plattenepithelkarzinom ein singulärer Leberbefall tendenziell häufiger war als bei Adenokarzinom. Bei Adenokarzinom traten im Vergleich multiple Metastasen tendenziell häufiger auf.

Die Analysen zur geschlechtsspezifischen Metastasenlokalisation erbrachten keine Auffälligkeiten. Aus diesem Grund wurde auf eine Darstellung dieser Daten verzichtet.

\section{Datenmanagement und Statistik}

Die Patientendaten wurden in eine ORACLE-basierte Datenbank durch Studienassistentinnen eingegeben. Das Datenmanagement und Plausibilitätsprüfungen folgten in halbjährlichen Intervallen während der Rekrutierung und der Nachbeobachtungszeit durch den Studienarzt. Implausibilitäten wurden den rekrutierenden und nachbeobachtenden Einrichtungen regelmäßig zurückgemeldet und entsprechende Fehler korrigiert. Fehlende Daten wurden durch Studienmitarbeiter aus den Krankenakten nachgetragen.

Die statistischen Berechnungen erfolgten mit dem Softwarepaket SPSS 11.0 am Institut für Medizinische Epidemiologie, Biometrie und Informatik der Martin-Luther-Universität Halle-Wittenberg. Die univariaten Überlebensanalysen erfolgten mittels KaplanMeier-Analyse, die multivariaten Analysen mittels Cox-Regression. Der Test für die Beurteilung der verschiedenen Metastasenlokalisationen war der Logrank-Test bzw. Chi-Quadrat-Test.

\begin{tabular}{|c|c|}
\hline \multicolumn{2}{|l|}{ Geschlecht und Alter } \\
\hline männlich & $280(82,6 \%)$ \\
\hline weiblich & $59(17,4 \%)$ \\
\hline Alter, männlich & $64,3(58,3-70,2)$ \\
\hline Alter, weiblich & $62,4(56,0-71,8)$ \\
\hline \multicolumn{2}{|l|}{ Leistungszustand } \\
\hline $0-1$ & $158(46,6 \%)$ \\
\hline 2 & $68(20,1 \%)$ \\
\hline $3-4$ & $113(33,3 \%)$ \\
\hline total & $339(100 \%)$ \\
\hline \multicolumn{2}{|l|}{ Histologie } \\
\hline großzelliges Karzinom & $7(2,1 \%)$ \\
\hline Plattenepithelkarzinom & $144(42,5 \%)$ \\
\hline Adenokarzinom & $75(22,1 \%)$ \\
\hline nichtkleinzellig o.n. A. & $113(33,3 \%)$ \\
\hline gesamt & $339(100 \%)$ \\
\hline
\end{tabular}

o. n. A. ohne weitere Angaben zur Histologie

Tab. 2 Metastasenlokalisation und Alter.

\begin{tabular}{|lll|}
\hline \multicolumn{1}{|c|}{$\mathbf{n ( \% )}$} & Alter \\
\hline Nebenniere & $27(8,0 \%)$ & $63,9(56,9-68,6)$ \\
\hline Hirn & $25(7,4 \%)$ & $57,8(54,7-62,8)$ \\
\hline Leber & $25(7,4 \%)$ & $70,2(62,4-73,0)$ \\
\hline Knochen & $25(7,4 \%)$ & $64,5(54,8-71,9)$ \\
\hline Lunge & $84(24,8 \%)$ & $66,3(60,6-71,6)$ \\
\hline mehrere & $122(36,0 \%)$ & $63,2(56,7-69,8)$ \\
\hline andere & $31(9,1 \%)$ & $63,6(56,3-69,4)$ \\
\hline gesamt & $339(100 \%)$ & $64,2(57,9-70,5)$ \\
\hline * p $<0,05$ (Chi-Quadrat-Test) & \\
\hline
\end{tabular}

Tab. 3 Metastasenlokalisationen in Abhängigkeit von der Histologie.

\begin{tabular}{|lccc|}
\hline & Plattenepithel-Ca & Adeno-Ca & NSCLC o.n. A. \\
\hline Nebenniere & $13(9 \%)$ & $8(11 \%)$ & $6(5 \%)$ \\
\hline Hirn & $9(6 \%)$ & $6(8 \%)$ & $10(8 \%)$ \\
\hline Leber & $14(10 \%)$ & $3(4 \%)$ & $8(7 \%)$ \\
\hline Knochen & $9(6 \%)$ & $7(9 \%)$ & $9(8 \%)$ \\
\hline Lunge & $39(27 \%)$ & $17(23 \%)$ & $28(23 \%)$ \\
\hline multiple & $46(32 \%)$ & $30(40 \%)$ & $46(38 \%)$ \\
\hline andere & $14(10 \%)$ & $4(5 \%)$ & $13(11 \%)$ \\
\hline gesamt & $144(100 \%)$ & $75(100 \%)$ & $120(100 \%)$ \\
\hline
\end{tabular}

keine signifikanten Unterschiede zwischen den Gruppen

\section{Ergebnisse}

\section{Überleben}

\section{Univariate Analyse}

Die Tab. 4 und $\bullet$ Abb. 1 demonstrieren das beobachtete Überleben in einer Kaplan-Meier-Analyse. Die Einjahresüberlebensrate unterscheidet sich in Abhängigkeit von der Metastasenlokalisation um 39 Prozentpunkte: Patienten mit Lungenmetastasen hatten eine Einjahresüberlebensrate von $44 \%$, während Patienten mit Lebermetastasen das schlechteste Outcome hatten mit $5 \%$ Einjahresüberleben.

Das beobachtete Überleben unterschied sich weder in Abhängigkeit von der Histologie noch in Abhängigkeit vom klinischen Leistungszustand. 
Tab. 4 Überleben, univariate Analyse (Kaplan Meier).

\begin{tabular}{|c|c|c|c|}
\hline & $\begin{array}{l}\text { Median } \\
\text { Monate }(95 \% \mathrm{Cl})\end{array}$ & 1-J-ÜLR & SE \\
\hline \multicolumn{4}{|l|}{ Lokalisation* } \\
\hline Nebenniere & $7,0(5,8-8,2)$ & $26,4 \%$ & 9,1 \\
\hline Hirn & $6,1(0,4-11,8)$ & $24,2 \%$ & 9,4 \\
\hline Leber & $2,2(0-4,8)$ & $4,8 \%$ & 4,6 \\
\hline Knochen & $6,4(4,5-8,2)$ & $18,5 \%$ & 9,2 \\
\hline Lunge & $11,1(7,9-14,2)$ & $44,5 \%$ & 5,8 \\
\hline andere & $7,0(0,5-13,2)$ & $30,8 \%$ & 9,0 \\
\hline mehrere & $3,8(2,9-4,7)$ & $15,4 \%$ & 3,5 \\
\hline \multicolumn{4}{|l|}{ Histologie } \\
\hline großzelliges Karzinom & $13,6(0-41,6)$ & $66 \%$ & 19 \\
\hline Adenokarzinom & $5,7(3,9-7,6)$ & $21 \%$ & 5,1 \\
\hline Plattenepithelkarzinom & $5,3(4,4-6,2)$ & $22 \%$ & 3,7 \\
\hline NSCLC o.n.A. & $6,8(5,9-7,8)$ & $30 \%$ & 4,8 \\
\hline \multicolumn{4}{|l|}{ Leistungszustand } \\
\hline $0-1$ & $6,4(4,8-8,0)$ & $28 \%$ & 4,4 \\
\hline 2 & $4,8(2,8-6,8)$ & $27 \%$ & 6,2 \\
\hline $3-4$ & $5,0(3,2-6,9)$ & $17 \%$ & 4,5 \\
\hline
\end{tabular}

NSCLC o. n. A.: nicht kleinzelliges Karzinom ohne weitere Spezifikation; 1-J-ÜLR: Einjahresüberlebensrate, SE: Standardfehler ${ }^{*} \mathrm{p}<0,05(\log$ rank $)$

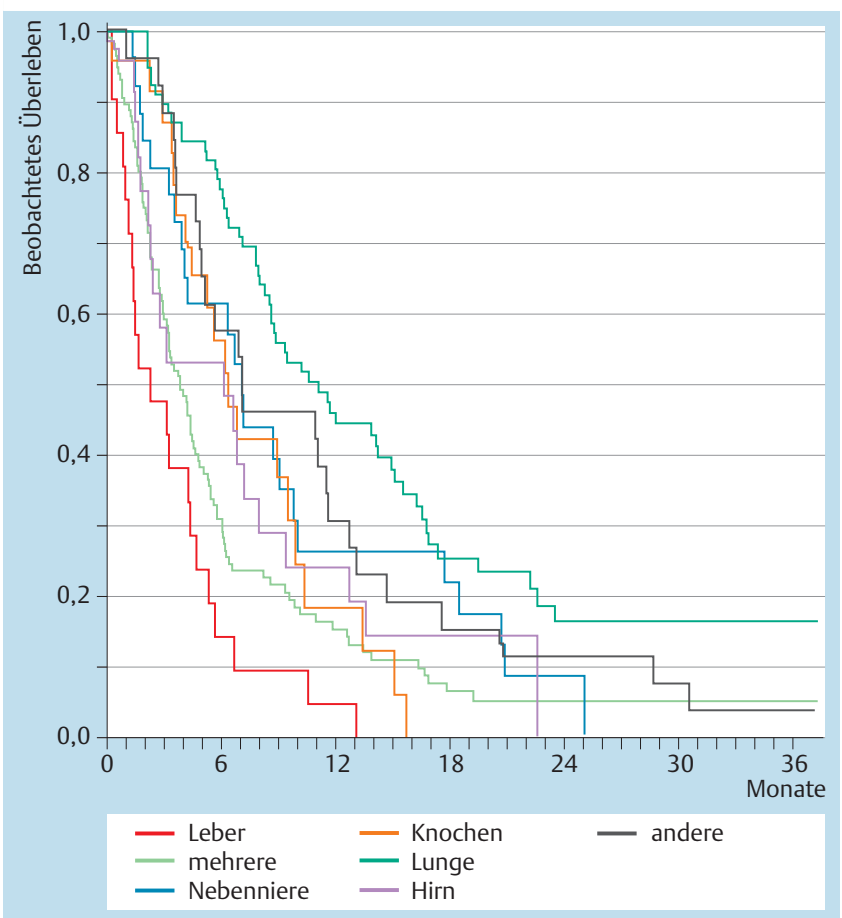

Abb. 1 Überleben in Abhängigkeit von der Metastasenlokalisation. $\mathrm{p}<0,05$ (log rank).

\section{Multivariate Analyse}

- Tab. 5 und $\odot$ Abb. 2 zeigen die Daten zur multivariaten Überlebensanalyse in Abhängigkeit von der Metastasenlokalisation. Als Kovariablen wurden das Alter, die Histologie, die Art der Therapie sowie der Performancestatus gewählt. Wir konnten zeigen, dass auch nach Adjustierung der genannten Kovariablen der prognostische Einfluss der Metastasenlokalisation bestätigt wurde. Die Einjahresüberlebenswahrscheinlichkeit ist $<5 \%$ bei Patienten mit Lebermetastasen. Die beste 1-Jahres-Überlebenswahrscheinlichkeit bestand mit etwa $47 \%$ bei Lungenmetastasen bzw. mit 33\% bei Nebennierenmetastasen.
Tab. 5 Überleben, multivariate Analyse (Cox Regression).

\begin{tabular}{|lllll|}
\hline & B & df & sign. & Exp(B) \\
\hline Alter & 0,018 & 1 & 0,336 & 1,01 \\
\hline $\begin{array}{l}\text { Chemotherapie } \\
\text { andere }\end{array}$ & 0,4 & 1 & 0,037 & 1,5 \\
\hline nur best supportive care & 0,73 & 1 & $<0,001$ & 2,07 \\
\hline PS & & 2 & 0,09 & \\
\hline PS 0-1 & ref & & & \\
\hline PS 2 & $-0,02$ & 1 & 0,907 & 0,98 \\
\hline PS 3-4 & 0,34 & 1 & 0,046 & 1,39 \\
\hline Metastasenlokalisation & & 6 & $<0,001$ & \\
\hline Nebenniere & ref & & & \\
\hline Hirn & 0,52 & 1 & 0,157 & 1,68 \\
\hline Leber & 1,32 & 1 & 0 & 3,74 \\
\hline Knochen & 0,38 & 1 & 0,327 & 1,46 \\
\hline Lunge & $-0,36$ & 1 & 0,217 & 0,69 \\
\hline mehrere & 0,65 & 1 & 0,016 & 1,92 \\
\hline andere & 0,41 & 1 & 0,214 & 1,51 \\
\hline Histologie & & 2 & 0,263 & \\
\hline Plattenepithel & ref & & & \\
\hline Adeno & 0,01 & 1 & 0,954 & 1,01 \\
\hline andere & $-0,24$ & 1 & 0,133 & 0,79 \\
\hline
\end{tabular}

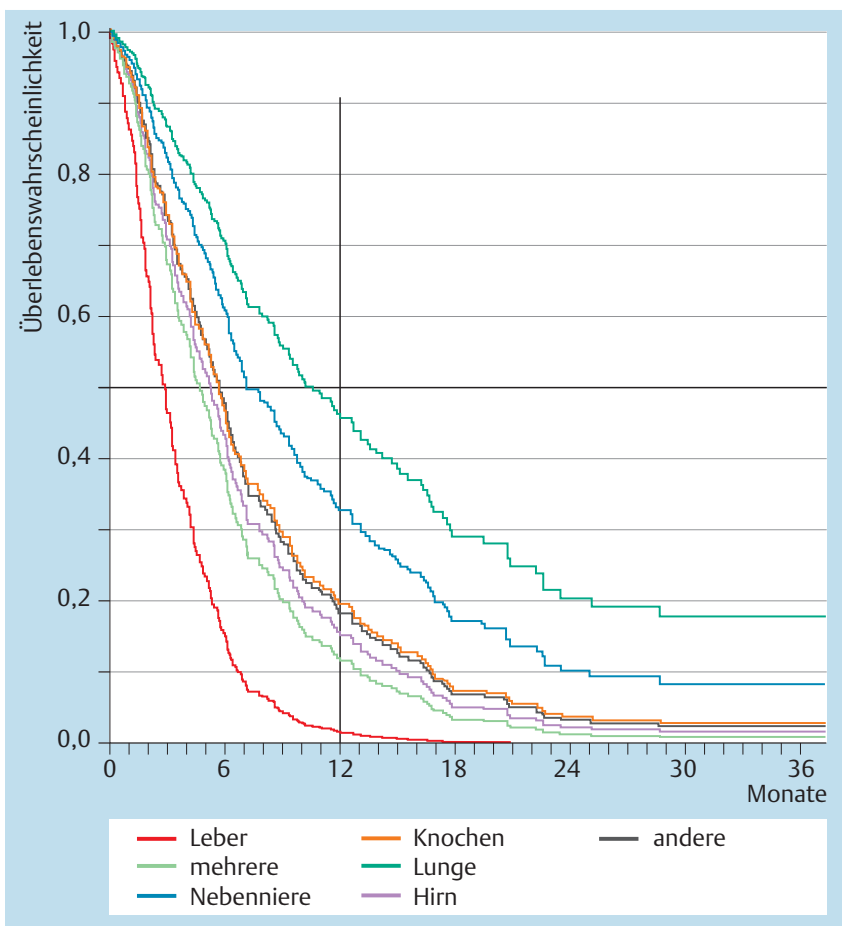

Abb. 2 Multivariate Überlebensanalyse (Cox Regression). Kovariable: Alter, Leistungszustand, Therapie, Histologie. ${ }^{*} p<0,05$.

Eine detaillierte Betrachtung der multivariaten Analyse zeigt, dass eine ungünstige Metastasenlokalisation einen stärkeren prognostischen Einfluss hat als beispielsweise die Therapieentscheidung hinsichtlich Chemotherapie oder best supportive care: Patienten mit Lebermetastasen hatten ein 3,7-fach erhöhtes Sterberisiko im Vergleich zu Patienten mit Nebennierenmetastasen, während Patienten, die nur best supportive care erhalten hatten, ein zweifach erhöhtes Sterberisiko im Vergleich zu chemotherapierten Patienten hatten. Auch ein schlechter klinischer Leistungszustand hat geringeren prognostischen Einfluss als die Metastasenlokalisation: Das Sterberisiko im Untersuchungszeit- 
Tab. 6 Einfluss klinischer Parameter auf die Therapieentscheidung.

\begin{tabular}{|c|c|c|}
\hline & $\begin{array}{l}\text { Chemotherapie } \\
\text { n (\%) }\end{array}$ & $\begin{array}{l}\text { keine Chemo- } \\
\text { therapie n (\%) }\end{array}$ \\
\hline \multicolumn{3}{|l|}{ Leistungszustand } \\
\hline $0-1$ & $60(48 \%)$ & $66(52 \%)$ \\
\hline 2 & $14(25 \%)$ & $42(75 \%)$ \\
\hline $3-4$ & $30(34 \%)$ & $57(66 \%)$ \\
\hline \multicolumn{3}{|l|}{ Histologie } \\
\hline großzelliges Karzinom & $6(86 \%)$ & $1(14 \%)$ \\
\hline Plattenepithelkarzinom & $48(33 \%)$ & $96(67 \%)$ \\
\hline Adenokarzinom & $33(44 \%)$ & $42(56 \%)$ \\
\hline nichtkleinzellig o.n.A. & $46(41 \%)$ & $67(59 \%)$ \\
\hline \multicolumn{3}{|l|}{ Metastasenlokalisation } \\
\hline Nebenniere & $11(41 \%)$ & $16(59 \%)$ \\
\hline Hirn & $12(48 \%)$ & $13(52 \%)$ \\
\hline Leber & $6(24 \%)^{*}$ & $19(76 \%)$ \\
\hline Knochen & $9(36 \%)^{*}$ & $16(63 \%)$ \\
\hline Lunge & $43(51 \%)$ & $41(49 \%)$ \\
\hline mehrere & $37(30 \%)^{*}$ & $85(70 \%)$ \\
\hline andere & $15(48 \%)$ & $16(52 \%)$ \\
\hline
\end{tabular}

raum war bei Patienten mit schlechtem klinischem Leistungszustand nur um den Faktor 1,4 erhöht im Vergleich zu den Patienten mit gutem klinischem Leistungszustand.

Alter und Histologie hatten auch in der multivariaten Analyse keine prognostische Relevanz.

\section{Versorgungsforschung: Einfluss klinischer Parameter auf die Therapieentscheidung \\ Insgesamt wurden 39\% der Patienten im Tumorstadium IV pri- mär mit Chemotherapie behandelt.}

Die Tab. 6 zeigt, dass in der hier dargestellten Studienpopulation der klinische Leistungszustand nur einen geringen Einfluss auf die Therapieentscheidung hat. 48\% der Patienten mit gutem klinischem Leistungszustand (PS 0 und 1) wurden chemotherapiert. Patienten in schlechtem Leistungszustand PS 3 und 4) wurden in 34\% d.F. einer Chemotherapie zugeführt.

Patienten mit Adenokarzinom bekamen in 44\% d. F. Chemotherapie und damit tendenziell, nicht signifikant, häufiger als Patienten mit Plattenepithelkarzinom. Der Anteil an chemotherapierten Patienten war bei Leber-, Knochen- und multiplen Metastasen signifikant geringer als bei den übrigen Lokalisationen.

\section{Diskussion}

$\nabla$

\section{1. Überleben}

In dieser Arbeit konnten wir in einer prospektiven Untersuchung zeigen, dass bei Patienten mit metastasiertem nicht kleinzelligem Bronchialkarzinom die Art der Metastasenlokalisation sowie die Anzahl der befallenen Organe prognostischen Einfluss haben. In einer retrospektiven Analyse von Jeremic et al. konnte in einer univariaten Analyse von zwei Chemotherapiestudien bei Patienten mit multiplen Metastasen sowie bei Patienten mit Lebermetastasen ähnliches medianes Überleben gezeigt werden [14]. Eine retrospektive Analyse mehrerer Chemotherapiestudien der South West Oncology Group (SWOG), die eine versus mehrere Metastasierungslokalisationen verglichen hat, kam zu einem ähnlichen Ergebnis [15].
Obwohl im Vergleich zu der Arbeit von Jeremic, der Patienten aus zwei Chemotherapiestudien retrospektiv analysierte, in der hier vorliegenden Untersuchung der Anteil an Patienten mit nur best supportive care $41 \%$ beträgt und somit deutlich höher ist, ist die Überlebensrate der Gesamtgruppe ähnlich. Eine Begründung dafür liegt vermutlich in der geringeren Aktivität der von Jeremic gewählten älteren Chemotherapieprotokolle (Carboplatin, Etoposid vs. Etoposid mono in Studie 1, Carboplatin, Etoposid po vs. Carboplatin, Etoposid iv in Studie 2). Diese These wird durch die retrospektive Analyse der SWOG unterstützt, die gezeigt hat, dass ältere Chemotherapieprotokolle mit schlechterem Überleben assoziiert waren als aktuellere [15].

Über die genannten Arbeiten hinausgehend, konnte in der hier vorliegenden Arbeit auch in der multivariaten Analyse gezeigt werden, dass die Metastasenlokalisation (unter Berücksichtigung des Einflusses von Alter, Art der Therapie, Leistungszustand und Histologie) signifikanten Einfluss auf die Prognose hat. Die genannten anderen klinischen Faktoren haben, multivariat analysiert, einen geringeren prognostischen Einfluss. Eine vergleichbare multivariate Analyse von Albain [15] konnte ebenso zeigen, dass eine multiple Metastasierung im Vergleich zu einer singulären Metastasierung mit einer schlechteren Überlebenswahrscheinlichkeit assoziiert war.

Vergleicht man die hier erreichten Überlebensraten mit den Daten der Literatur, so konnte gezeigt werden, dass das Überleben in der Routineversorgung ähnlich dem Überleben in den Studiendaten ist: Das hier erreichte gute Überleben der Patienten mit pulmonalen Metastasen ist vergleichbar mit Daten einer retrospektiven Subgruppenanalyse von Patienten mit histologisch gesicherten pulmonalen Metastasen von Nakajima et al. [16]. Gerade der pulmonale Rundherd, ob als Primärtumor oder als Metastase, wurde auch in der aktuellen S3-Leitlinie der Deutschen Gesellschaft für Pneumologie und Beatmungsmedizin und der Deutschen Krebsgesellschaft einer neuen Bewertung unterzogen [28]. Es wurde in der deutschen Leitlinie die hier dargestellte gute Prognose dieser Patientengruppe neu bewertet, indem der ipsilaterale Herd im nicht primärtumortragenden Lappen nicht mehr als M1, sondern als T4 bewertet wurde [28]. Die Einjahresüberlebensrate von Patienten mit Hirnmetastasen war in einer Arbeit von Jacot [17] 25\% und entspricht in etwa unseren Daten. Hinsichtlich des Überlebens mit Nebennierenmetastasen gibt es eine kleine Arbeit von Luketich [18], der ein mittleres Überleben zwischen 8,5 Monaten unter Chemotherapie und 31 Monaten nach Chemotherapie und chirurgischer Operation beschreibt. Die Patienten der hier dargestellten Untersuchung erhielten insgesamt nur in $41 \%$ d.F. Chemotherapie. Eine multimodale Behandlung unter Einbeziehung der Adrenalektomie wurde in unserem Krankengut nicht dokumentiert. Insofern ist das mediane Überleben von 7 Monaten vergleichbar mit den Daten der nicht operierten Patienten dieser Arbeit [18].

\section{Versorgungsforschung}

Der versorgungsepidemiologische Teil dieser Arbeit zeigt, dass in der Studienregion im untersuchten Zeitraum Patienten mit metastasiertem nicht kleinzelligem Bronchialkarzinom zu unter $50 \%$ einer Chemotherapie zugeführt werden. Daneben ist auffällig, dass der klinische Leistungszustand nur geringen Einfluss auf die Therapieentscheidung zur Chemotherapie hatte. Insbesondere ist verwunderlich, dass Patienten in gutem klinischem Leistungszustand nur in $48 \%$ der Fälle Chemotherapie erhalten haben. Dies zeigt, dass sowohl die nationalen $[12,28]$ als auch die internationalen Empfehlungen [19] in der Region im Untersu- 
chungszeitraum nicht konsequent umgesetzt waren. Wenngleich die Diskrepanz zu den Empfehlungen erheblich scheint, zeigt der Vergleich mit anderen regionsbezogenen Daten einen ähnlichen Durchsetzungsgrad der Therapieempfehlungen. In der Arbeit von Mc Gregor et al. [10] war der Anteil von Patienten mit nur best supportive care an allen Patienten mit Bronchialkarzinom 43\%. Diese Behandlungsrealität wird unter anderem durch den Patientenwunsch und durch Versorgungsstrukturen bedingt. Die best-supportive-care-Raten schwanken einrichtungsbezogen zwischen Kliniken, die überwiegend an klinischen Studien teilnehmen, und Kliniken, die sich seltener beteiligen, um 34 Prozentpunkte (17-51\%). Dies widerspiegelt auch ein entsprechendes Zuweisungsverhalten und ist nicht per se ein Qualitätsdefizit einer einzelnen Einrichtung oder Region.

Bei Patienten mit Plattenepithelkarzinom wurde Chemotherapie tendenziell seltener angewandt als bei Patienten mit Adenokarzinom. Die Therapieentscheidung bei Patienten mit Großzelligem Karzinom kann aufgrund der geringen Fallzahl nicht bewertet werden. Aus der Analyse der Daten der Literatur gibt es bisher keine Hinweise darauf, dass die Histologie einen Einfluss auf die Entscheidung für oder gegen eine aktive Therapie bei metastasiertem NSCLC hat.

Während die Chemotherapieraten bei allen anderen singulären Metastasenlokalisationen zwischen 41 und 51\% liegen, wurde bei Knochenmetastasen, Lebermetastasen und multiplen Metastasen Chemotherapie signifikant seltener angewandt. Diese Therapieentscheidung ist nicht durch einen erhöhten Anteil an Patienten mit schlechtem Leistungszustand in diesen Gruppen bedingt. In der Literatur gibt es bisher keine Hinweise darauf, dass die Effektivität der Chemotherapie bei Knochenmetastasen oder bei multiplen Metastasen schlechter ist als bei anderen Metastasenlokalisationen. Hier wird nach Ansicht der Autoren ein Therapieangebot ohne eine entsprechende Datengrundlage empirisch begrenzt.

\section{Diskussion der Methodik}

Die großen Überlebensunterschiede der verschiedenen Metastasenlokalisationen lassen einen Bias oder eine in dieser Arbeit nicht erfasste unterschiedliche Tumorbiologie vermuten. Sowohl bezogen auf einzelne Metastasenlokalisationen [13,15-17] als auch im Vergleich singuläre versus multiple Metastasierung [16] sind die hier dargestellten Überlebensdaten jedoch mit den Daten aus kontrollierten klinischen Studien vergleichbar.

Die Limitationen dieser Studie liegen ebenso in dem hohen Anteil nicht weiter spezifizierter nicht kleinzelliger Histologie. Die Wahrscheinlichkeit, dass an dieser Stelle der Effekt einer Co-Variablen nicht abgebildet wird, halten die Autoren jedoch für gering, da es im Vergleich zwischen Adenokarzinom und Plattenepithelkarzinom in dieser Untersuchung keine histologieabhängige Organotropie gibt. Auffällig ist das niedrige Alter der Patienten mit Hirnmetastasen. Die prognostischen Unterschiede bestehen jedoch nicht nur hinsichtlich der Hirnmetastasen, sondern auch der anderen Lokalisationen. Die unterschiedliche Häufigkeit an Chemotherapie in Abhängigkeit von der Metastasenlokalisation hat in der univariaten Analyse sicher große prognostische Bedeutung. Der prognostische Effekt der Chemotherapie wird nach Meinung der Autoren durch die multivariate Analyse (CoxRegression) korrigiert. Diese Ergebnisse, die auf der Anwendung eines statistischen Testverfahrens beruhen, sollten letztendlich durch eine entsprechend konzipierte Studie bestätigt werden. Eine differenzierte Darstellung der Überlebensdaten, bezogen auf die jeweilige Histologie und Lokalisation, ist in Anbetracht der dann resultierenden kleinen Gruppen nicht sinnvoll. Eine andere Limitation dieser Untersuchung ist die Unterschätzung des Metastasierungsgrades. Entsprechend der aktuellen Empfehlungen ist mit der beschriebenen Methodik der Metastasensuche das Risiko der Unterschätzung der Metastasierung relativ gering [20,21].

Aktuelle PET-Studien [22,23] zeigen, dass eine zusätzliche Positronen-Emissions-Tomografie okkulte Metastasen in etwa 10 bis $20 \%$ der Fälle anzeigen kann. Bei fehlenden klinischen Zeichen bzw. den in der Methodik beschriebenen negativen Befunden ist die Wahrscheinlichkeit, okkulte Metastasen zu finden so gering, dass weitere Prozeduren nicht empfohlen werden [20,21,24]. Eine weitere Fehlermöglichkeit ist die Überschätzung einer Metastasierung infolge nicht konsequenter morphologischer Sicherung. Dies würde in dieser Untersuchung zu einer Änderung des Überlebens durch Ausschluss falsch negativer Befunde führen. Die einzigen in dieser Hinsicht infrage kommenden Lokalisationen sind die Lunge [20], die Nebennieren [25,26] und die multiple Metastasierung. Für die Sicherung von Nebennierenmetastasen galt der folgende Algorithmus: Bei Auffälligkeit im CT, Klärung durch MRT; wenn unsicher, dann histologische Sicherung notwendig. Lungenmetastasen wurden histologisch gesichert, wenn sie eine therapeutische Relevanz hatten, dies war in jedem Fall bei Patienten mit alleinigen Lungenmetastasen gegeben. Das schlechte Überleben von Patienten mit multiplen Metastasen spricht auch gegen einen relevanten Anteil an falsch positiven multiplen Metastasen.

\section{Zusammenfassung}

$\nabla$

In dieser prospektiven versorgungsepidemiologischen Arbeit konnten wir zeigen, dass das Überleben bei metastasiertem nicht kleinzelligem Bronchialkarzinom wesentlich von Art und Umfang der Metastasenlokalisation bestimmt wird. Der prognostische Einfluss des klinischen Leistungszustandes ist im Vergleich zum Einfluss der Metastasenlokalisation geringer. Wenngleich diese Arbeit aufgrund des versorgungsepidemiologischen Ansatzes methodische Limitationen hat, sollte vor dem Hintergrund dieser Ergebnisse bei der Planung von Chemotherapiestudien über eine Stratifizierung nach der Metastasenlokalisation nachgedacht werden. Aus der unterschiedlichen Prognose eine Therapieentscheidung abzuleiten ist nicht gerechtfertigt.

Unter dem Teilaspekt der regionalen externen Qualitätssicherung ist festzustellen, dass im Untersuchungszeitraum die Chemotherapierate bei Patienten mit metastasiertem Bronchialkarzinom im Vergleich zu den Leitlinienempfehlungen gering war, dies aber anderen epidemiologisch gewonnenen Daten der Literatur entspricht. Andere regionale Besonderheiten der Versorgung von Bronchialkarzinompatienten konnten durch diese Analysen aufgezeigt und mit der Literatur verglichen werden.

Diese Arbeit zeigt neben dem Aspekt der prognostischen Heterogenität der Patienten im klinischen Tumorstadium IV des nicht kleinzelligen Bronchialkarzinoms die aktuelle Behandlungsrealität im südlichen Sachsen-Anhalt auf. Diese unterscheidet sich von den bisher publizierten Daten kontrollierter klinischer Studien selektierter Patientengruppen deutlich. Weitere Analysen dieser Art sind sinnvoll, um regionale Qualitätssicherung zu betreiben und die Behandlungsrealität mit den Daten kontrollierter Studien zu vergleichen. 


\section{Kooperierende Abteilungen/Danksagung}

Wir bedanken uns bei den folgenden kooperierenden

Abteilungen:

Klinik für Herz- und Thoraxchirurgie der

Martin-Luther-Universität Halle-Wittenberg,

Direktor Prof. Dr. R. Silber

Chirurgische Klinik des Diakoniewerks Halle,

Herrn Oberarzt O. Fischbeck

Lungenklinik Ballenstedt,

Herrn Chefarzt Dr. F. Fischer

Innere Klinik des Diakoniewerks Halle,

Herrn Chefarzt Dr. R. Heine

Klinik für Innere Medizin II der Martin-Luther-Universität

Halle-Wittenberg,

Frau Oberärztin Dr. B. Wollschläger

Krankenhaus Dessau-Alten,

Herrn Oberarzt PD Dr. J. Schreiber

Strahlenklinik der Martin-Luther-Universität Halle-Wittenberg,

Herrn Prof. Dr. J. Dunst

Praxis für Strahlentherapie Halle,

Herrn Privatdozent Dr. K. Heider

Weiterhin nahmen an dieser Studie 24 regionale Krankenhäuser, alle Pneumologen, Onkologen und viele Allgemeinpraktiker teil.

Die Studie wurde vom Bundesministerium für Gesundheit geför-

dert,

FKZ FB 2-43332-70/8.

\section{Interessenkonflikt}

\section{$\nabla$}

Die Autoren geben an, dass kein Interessenkonflikt besteht.

\section{Literatur}

1 Parkin M, Pisani P, Ferlay J. Global cancer statistics. Cancer J Clin 1999; 49: $33-64$

2 Reis LAG, Kosary CL, Hankey BF et al. SEER cancer statistics review, 1973 - 1996. Bethesda, MD: National Cancer Institute, 1999

3 Ihde DC. Chemotherapy of Lung Cancer. N Engl J Med 1992; 327: 1434 1441

4 DeVita VT, Hellman S, Rosenberg SA. Cancer: principles and practice of oncology. Philadelphia, PA: J.B. Lippincott, 1993

5 Bonomi P, Kim K, Fairclough D, Cella D et al. Comparison of survival and quality of life in advanced non-small-cell lung cancer patients treated with two dose levels of paclitaxel combined with cisplatin versus etoposide with cisplatin: results of an Eastern Cooperative Oncology Group trial. J Clin Oncol. 2000; 18: 623-31

6 Ries L, Elsner M, Kosary C. Cancer statistics review 1975-2002. Bethesda: MD National Cancer Institute, 2005

7 Scagliotti GV, Parikh P, Pawel J von et al. Phase III study comparing cisplatin plus gemcitabine with cisplatin plus pemetrexed in chemotherapy-naive patients with advanced-stage non-small-cell lung cancer. J Clin Oncol 2008; 26: 3543-3551

8 Shaw GL, Gazdar AF, Phelps R et al. Correlation of in vitro drug sensitivity testing results with response to chemotherapy and survival: comparison of non-small cell lung cancer and small cell lung cancer. J cell biochem 1996; 24: 173-185

9 Coebergh JWW, Janssen-Heijnen MLG, Louwman WJ et al. Cancer incidence, care and survival. Eindhoven Cancer Registry (IKZ), 2001
10 Gregor A, Thomson CS, Brewster DH et al. Management and survival of patients with lung cancer in Scotland diagnosed in 1995: results of a national population based study. Thorax 2001; 56: 212-217

11 Miller VA, Kris MG et al. Bronchioloalveolar Pathologic Subtype and Smoking History Predict Sensitivity to Gefitinib in Advanced NonSmall-Cell Lung Cancer. J Clin Oncol 2004; 22: 1103-1109

12 Thomas M, Baumann M, Deppermann $M$ et al. Empfehlungen zur Therapie des Bronchialkarzinoms. Gemeinsame Empfehlung der Deutschen Gesellschaft für Pneumologie (DGP), der Deutschen Gesellschaft für Radioonkologie (DEGRO), der Deutschen Gesellschaft für Thoraxchirurgie (DGT) und der Arbeitsgemeinschaft Internistische Onkologie in der Deutschen Krebsgesellschaft (AIO). Pneumologie 2002; 56: $113-131$

13 Shepherd FA. Treatment of advanced non-small cell lung cancer. Semin in oncol 1994; $21: 7-18$

14 Jeremic BA, Dagovic BA, Nikolic NA. Pretreatment clinical prognostic factors in patients with stage IV non-small cell lung cancer (NSCLC) treated with chemotherapy. J Cancer Res Clin Oncol 2003; 129: 114122

15 Albain KS, Crowley JJ, LeBlanc M, Livingston RB. Survival determinants in extensive-stage non-small-cell lung cancer: the Southwest Oncology Group experience. J Clin Oncol 1991; 9: 1618-1626

16 Nakajima , Furuse A, Oka T, Kohno T et al. Excellent Survival in a Subgroup of Patients With Intrapulmonary Metastasis of Lung Cancer. Ann Thorac Surg 1996; 61: 158-163

17 Jacot W, Quantin X, Boher J-M et al. Brain metastases at the time of presentation of non-small cell lung cancer: a multi-centric AERIO* analysis of prognostic factors. British Journal of Cancer 2001; 84: 903-909

18 Luketich JL, Burt ME. Does Resection of Adrenal Metastases From NonSmall Cell Lung Cancer Improve Survival? Ann Thorac Surg 1996; 62: $1614-1616$

19 ASCO Special Article: American society of clinical oncology treatment of unresectable Non-small cell lung cancer guideline: update 2003. J Clin Oncol 2004; 22: 330-353

20 Schiller JH. Current Standards of Care in Small-Cell and Non-Small. Cell Lung Cancer. Oncology 2001; 61: 3-13

21 Silvestri GA, Tanoue LT, Margolis ML et al. The Non-invasive Staging of Non-small Cell Lung Cancer The Guidelines. CHEST 2003; 123: 147 156

22 Pieterman RM, van Putten JWG, Meuzelaar JJ et al. Preoperative staging of non-small-cell lung cancer with positronemission tomography. N Engl J Med 2000; 343: 254-261

23 Saunders CAB, Dussek JE, O'Doherty MJ et al. Evaluation of fluorine-18fluorodeoxyglucose whole body positron emission tomography imaging in the staging of lung cancer. Ann Thorac Surg 1999; 67: 790 - 797

24 British Thoracic Society, Society of Thoracic Surgeons of Great Britain, Ireland Working Party. Guidelines on the selection of patients with lung cancer for surgery. Thorax 2001; 56: 89-106

25 Schwartz LH, Ginsberg MS, Burt ME et al. MRI as alternative to CT guided biopsy of adrenal masses in patients with lung cancer. Ann Thorac Surg 1998; 65: 193-197

26 Porte HL, Ernst OJ, Delebecq T et al. Is computed tomography guided biopsy still necessary for the diagnosis of adrenal masses in patients with resectable non-small cell lung cancer? Eur J Cardio Thorac Surg 1999; 15: 597-601

27 Tammemagi CM, Neslund-Dudas C, Simoff $M$ et al. In lung cancer patients, age, race-ethnicity, gender and smoking predict adverse comorbidity, which in turn predicts treatment and survival. J Clin Epidemiol 2004; 57: 597-609

28 Goeckenjan G, Sitter H, Thomas M et al. Prävention, Diagnostik, Therapie und Nachsorge des Lungenkarzinoms. Interdisziplinäre S3-Leitlinie der Deutschen Gesellschaft für Pneumologie und Beatmungsmedizin und der Deutschen Krebsgesellschaft. Pneumologie 2010; 64: S23S156 